\title{
Nivolumab in chemotherapy-resistant cervical cancer: report of a vulvitis as a novel immune-related adverse event and molecular analysis of a persistent complete response
}

\author{
Florence Baettig ${ }^{1}$, Tatjana Vlajnic ${ }^{2}$, Marcus Vetter ${ }^{3}$, Katharina Glatz², Jürgen Hench², Stephan Frank², Michel Bihl²,
} Roberto Lopez ${ }^{1,3}$, Michael Dobbie ${ }^{4}$, Viola Heinzelmann-Schwarz ${ }^{3,5}$ and Céline Montavon ${ }^{3,5^{*}}$ (D)

\begin{abstract}
Background: Treatment options for advanced cervical cancer are limited and patients experiencing recurrence after first-line cisplatin-based chemotherapy and bevacizumab have a poor prognosis. A recent phase II study in advanced cervical cancer has demonstrated a disease control rate of $68.4 \%$ with the immune checkpoint inhibitor nivolumab. By blocking immune checkpoints, immunotherapy puts the immune system into a state of hyperactivation that can cause immune-related adverse events.

We present the clinical, pathological and molecular data of a patient with metastatic cervical cancer and progressive disease after second-line therapy. We report on the therapeutic response under third-line immunotherapy with nivolumab, the immune-related adverse events (IRAE), and their successful management.

Case presentation: We report the case of a 62-year-old woman who was diagnosed with advanced squamous cell carcinoma of the cervix with paraaortic lymph node metastases. After an initial combined radio-chemotherapy with cisplatin, she developed local and nodal (supraclavicular) recurrence. Second-line chemotherapy with 6 cycles of carboplatin, paclitaxel, and bevacizumab resulted in a partial response for 6 months. Checkpoint inhibition with nivolumab was started due to progression, leading to persistent complete remission.

Immunotherapy was well tolerated for 8 months until the patient presented with an immune-related isolated vulvitis, which was successfully managed with topical corticosteroids.

Conclusions: The persistent complete response after third-line treatment for relapsed chemotherapy-resistant cervical cancer presented in this case highlights the potential of immunotherapy for patients with advanced cervical cancer impressively. To our knowledge, this is the first report of an isolated immune-related vulvitis under nivolumab. This adverse event might be underdiagnosed and mistreated, however, it is of importance due to its impact on quality of life, sexual wellbeing and compliance of patients. Successful IRAE management may enable prolonged immune checkpoint inhibitor therapy. In the future, routine molecular tumour profiling is likely to aid in the stratification of cenvical cancer patients for immunotherapy. Here, we provide the methylome data of a case with complete response.
\end{abstract}

Keywords: Immunotherapy, Nivolumab, Immune-related adverse event, Vulvitis, Cervical cancer therapy, Chemotherapy, DNA methylation profiling, Human papillomavirus, Tumour mutational burden, Copy number profile

\footnotetext{
* Correspondence: celine.montavon@usb.ch

${ }^{3}$ Gynaecological Cancer Centre, University Hospital Basel and University of Basel, Basel, Switzerland

${ }^{5}$ Hospital for Women, Gynaecological Oncology, University Hospital Basel,

Spitalstrasse 21, 4031 Basel, Switzerland

Full list of author information is available at the end of the article
}

(c) The Author(s). 2019 Open Access This article is distributed under the terms of the Creative Commons Attribution 4.0 International License (http://creativecommons.org/licenses/by/4.0/), which permits unrestricted use, distribution, and reproduction in any medium, provided you give appropriate credit to the original author(s) and the source, provide a link to the Creative Commons license, and indicate if changes were made. The Creative Commons Public Domain Dedication waiver (http://creativecommons.org/publicdomain/zero/1.0/) applies to the data made available in this article, unless otherwise stated. 


\section{Background}

Immunotherapy with immune checkpoint inhibitors has emerged as a novel option for many patients with advanced cancers whom previously had limited treatment options and experienced poor outcomes. While clinical studies have demonstrated survival benefits and durable responses in various cancer entities, immunotherapy of gynaecological cancers is still in relative infancy. In the United States, pembrolizumab, an antibody against programmed cell death protein 1 (PD-1), is approved for advanced endometrial cancers with high levels of microsatellite instability (MSI-high) and for recurrent or progressive metastatic cervical cancer positive for programmed death-ligand 1 (PD-L1), suggesting that these parameters could serve as predictive biomarkers.

Under physiological conditions, immune checkpoints play a crucial role in preventing autoimmunity [1]. Through the expression of PD-L1, cancer cells modulate the immune checkpoint to downregulate $\mathrm{T}$ cells, thereby protecting themselves from immune attack [2]. Immune checkpoint inhibitors reduce the interaction between cancer cells and $\mathrm{T}$ cells so that re-activated lymphocytes can destroy their malignant targets. Antibodies against PD-1 such as nivolumab have recently been approved for the treatment of different tumour types, particularly melanoma and non-small cell lung cancer [2].

More than $95 \%$ of cervical cancers are caused by a human papillomavirus (HPV) infection. Despite Papanicolaou testing, HPV screening, and prophylactic HPV vaccination, cervical cancer remains the fourth most common cause of death by cancer in women worldwide. The 5-year overall survival (OS) of recurrent or metastatic cervical cancer is poor (around 15\%), mainly due to limited treatment options.

For the last 20 years, the standard of care for patients with recurrent or metastatic cervical cancer has been cisplatin-based chemotherapy combined with paclitaxel. However, despite the addition of other agents such as vinorelbine, gemcitabine or topotecan [3], most patients deteriorated rapidly, developing platinum resistance at recurrence [4]. Adding bevacizumab, an anti-vascular endothelial growth factor antibody, to chemotherapy became the standard of care since the Gynecologic Oncology Group 240 trial demonstrated a survival benefit of almost 4 months [5]. Recent evidence indicates the potential usefulness of immune checkpoint inhibitors in cervical cancer [4] with objective response rates (ORR) in recurrent and/or advanced cervical cancer ranging from 12.2 to $26 \%$ [6]. However, it remains challenging to select patients with a potential therapeutic response and to anticipate the magnitude of response. Predictive markers of therapeutic response to immunotherapy are still unclear. Increased PD-L1 expression by tumour and immune cells as well as elevated tumour mutational burden (TMB; mutations per tumour genome coding region) have been associated with an increased likelihood of response [7]. High-TMB tumours tend to have more neoantigens serving as potential targets for the immune system [8]. Furthermore, patients with microsatelliteunstable (MSI-high) tumours seem to qualify for immunotherapy regardless of tumour type [9]. Last, immune responsiveness signatures based on methylation profiles, as already identified in lung cancer, are rapidly moving into clinical focus [10].

Whereas immune checkpoints are crucial in maintaining self-tolerance, their inhibition by immunotherapy puts the immune system into a hyperactive state that can cause immune-related adverse events (IRAE). IRAEs are frequent and occur in up to $70 \%$ of patients treated with anti-PD1/PD-L1 antibodies. Typically, IRAEs occur within weeks to a few months after treatment initiation, yet, reports on delayed IRAEs one year after treatment discontinuation have also been recorded [11]. Any organs can be affected by an IRAE, which can, in some cases, be life-threatening or even lethal [11].

We report a patient with persistent complete response after third-line treatment with Nivolumab for relapsed chemotherapy-resistant, high-TMB cervical cancer, which underlines the potential of immunotherapy. In addition, we describe vulvitis as an isolated IRAE under nivolumab, which was successfully treated with topical corticosteroids.

\section{Case presentation}

A 62-year-old woman was diagnosed with squamous cell carcinoma of the cervix uteri FIGO Stage IIIC2(r) (according to FIGO 2018). Computed tomography (CT) at first diagnosis showed a large cervical mass of $9 \mathrm{~cm}$, enlarged iliac and paraaortic lymph nodes, and right hydronephrosis. Prior personal and family history were negative. First-line treatment included combined radiochemotherapy with 6 cycles of cisplatin $40 \mathrm{mg} / \mathrm{m}^{2}$ weekly and lymph node irradiation, which was followed by intra-cervical brachytherapy with Iridium-192 resulting in a partial response. Three months later, progressive disease with a new palpable mass in a left supraclavicular lymph node and suspicion of pulmonary metastases (small nodules of $6 \mathrm{~mm}$, not amenable to biopsy) was noted. A mixed response of the pelvic nodal metastases as well as a progression of the cervical mass was documented. After radiotherapy of the left supraclavicular lymph node metastasis and second-line chemotherapy with 6 cycles of carboplatin/AUC6, paclitaxel $175 \mathrm{mg} /$ $\mathrm{m}^{2}$, and bevacizumab $15 \mathrm{mg} / \mathrm{kg}$, partial response (regression of all lesions without complete resolution) for 6 months was achieved. However, bevacizumab had to be interrupted due to rectorrhagia and ensuing anaemia. Due to progressive disease with new retrocrural and 
paraesophageal lymph node metastases, local radiotherapy was applied and a third-line systemic treatment with nivolumab ( $3 \mathrm{mg} / \mathrm{kg}$ q2 $\mathrm{w}$ for 17 months, then according to Checkmate-358 with $240 \mathrm{mg} \mathrm{q} 2 \mathrm{w}$ ) was initiated leading to complete remission as verified by positron emission tomography-computed tomography (PET-CT) at 12 months after immunotherapy initiation. Complete remission was also documented after 22 months of treatment.

The immunotherapy was well tolerated over the course of 8 months, then the patient presented with a well-delimited inflammation of the vulva and perianal region with ulceration and epidermolysis (Fig. 1a). A bacterial, fungal, or viral infection was excluded by microbiological analyses. Histology of a punch biopsy revealed a lichenoid interface dermatitis with a pronounced lymphocytic infiltrate along the dermoepidermal junction as well as intraepithelial. Immunohistochemically, most of lymphocytes were CD8-positive T cells intermixed with CD4-positive T cells (Fig. 2), consistent with an immunotherapy-related vulvitis. This IRAE was successfully treated by topical corticosteroid (clobetasol propionate 0.05\%, 1-2x daily; Fig. 1b).

We performed molecular analyses of the primary tumour. The tumour was positive for PD-L1 (clone SP263, Ventana), as $30 \%$ of tumour infiltrating immune cells were PD-L1 positive (defined as the proportion of tumour area occupied by PD-L1 staining of any intensity in immune cells), whereas the tumour cells were PD-L1 negative. Unexpectedly, the tumour was negative for p16 by immunohistochemistry, so that an HPV-association appeared unlikely. A qPCR-based HPV test (Anyplex ${ }^{\mathrm{Ta}}$ II HPV28 Detection, Seegene) on DNA from formalinfixed, paraffin-embedded tissue was negative. To further characterize the neoplasm, we performed a genomewide DNA methylation analysis (Infinium Methylation EPIC, Illumina) and compared the methylation pattern by a combined filtering and dimension reduction approach $[12,13]$ with reference data on various cancer types (The Cancer Genome Atlas, TCGA) including $\mathrm{HPV}$-associated cervical squamous cell carcinomas, as specific HPV-associated structural aberrations and increased target-gene expression have been reported [14]. We observed the highest concordance with the methylation classes of squamous cell carcinomas, particularly of the cervix, head and neck, and oesophagus (Fig. 3a. Methylation array data were also analysed for copy number changes, revealing a relatively flat profile (Fig. 3b,c).

TMB was exceptionally high with 44 mutations/megabase (Oncomine ${ }^{\mathrm{Tm}}$ Tumor Mutation Load Assay, Thermo Fisher Scientific), even though cut-off levels for nonHPV-related cervical cancers have yet to be defined. The $\mathrm{TMB}$ sequencing data were further screened for diseaserelated somatic variants. They revealed a pathogenic mutation in the PIK3CA gene and a likely pathogenic mutation in the $E R B B 2$ gene with allelic frequencies close to $40 \%$, matching the estimated tumour proportion of $80 \%$ ). Somatic mutations in both of these genes have been suggested to play a role in the pathogenesis of cervical squamous cell carcinoma [15].

Immunohistochemistry for DNA damage repair proteins (MLH1, MSH2, MSH6, PMS2) showed preserved expression of all examined proteins, consistent with a microsatellite stable (MSS) carcinoma.

\section{Discussion}

We report on a patient with primary advanced cervical cancer with paraaortic lymph node metastases, which

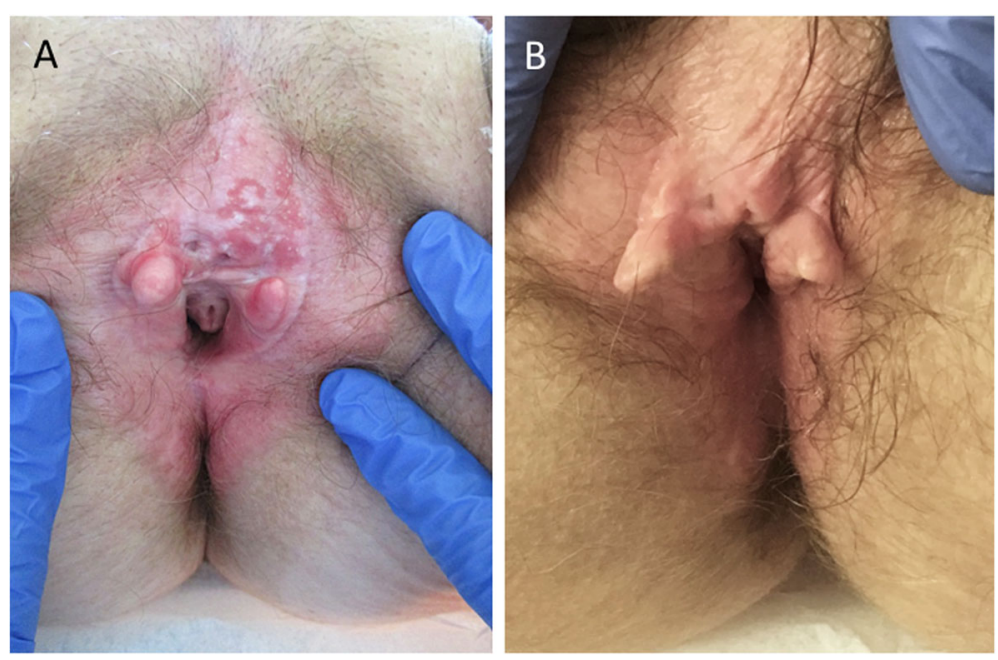

Fig. 1 Clinical appearance and successful treatment of the IRAE. a Ulcerative vulvitis six months after initial IRAE symptoms; biopsy shown in Fig. 2 was taken. $\mathbf{b}$ Successful management of IRAE with topical corticosteroids 

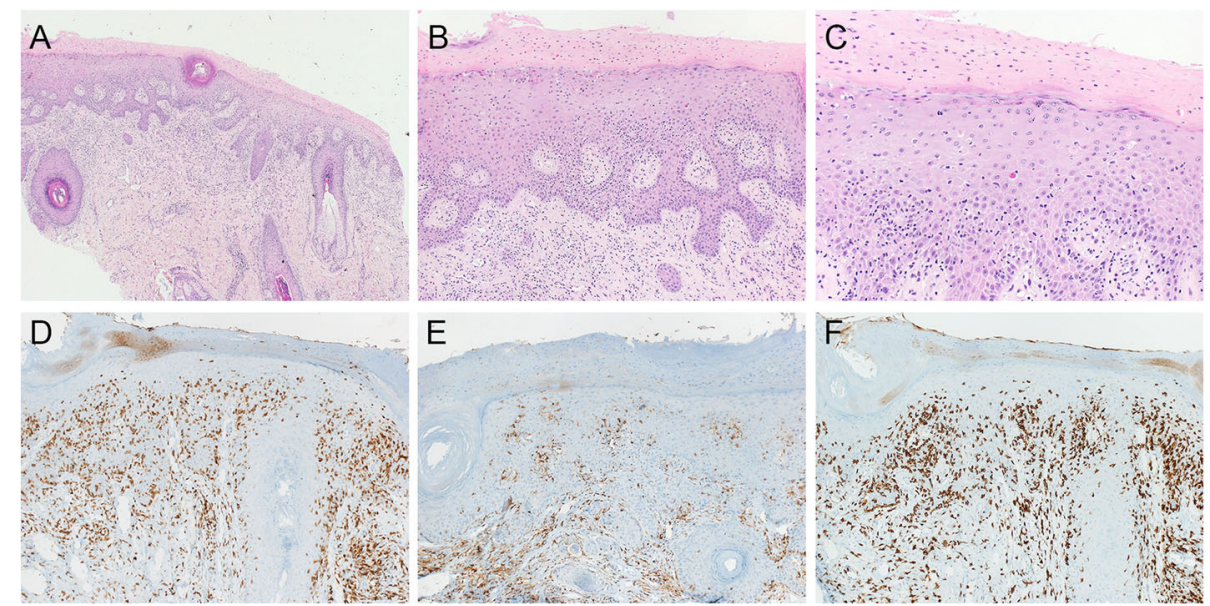

E

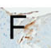

Fig. 2 a-c Biopsy of the vulva with lichenoid interface dermatitis: Squamous epithelium with hyper- and parakeratosis, irregular acanthosis, and dense band-like and perivascular lymphocytic infiltrate along the dermo-epidermal junction as well as intraepithelial lymphocytic infiltrate. Scattered necrotic keratinocytes (original magnification a 40x, b 100x, c, 200x). e-f Immunohistochemistry for T cell markers CD3, CD4, and CD8: Intraepithelial T cells predominantly express CD8 (original magnification e, f 100x)

developed a complete and persistent remission under third-line therapy with nivolumab. Immunotherapy with checkpoint inhibitors is an emerging option for many types of solid cancers, including advanced cervical cancer for which data remain limited [6].

The PD-1/PD-L1 pathway is one of the most widely understood immune mechanisms involved in cancer, including in cervical carcinoma. PD-L1 expression has been reported in $95 \%$ of cervical intraepithelial neoplasms and $80 \%$ of squamous cell carcinomas while it was absent in normal cervical mucosa [1]. Persistent HPV infections are known to be involved in cervical carcinogenesis and to correlate with a significant PDL1 up-regulation in tumour cells [16]. Checkmate-358 is a phase I/II trial investigating the response to nivolumab in HPV-associated advanced cervical $(n=19)$ as well as vaginal and vulvar $(n=5)$ cancers [17]. The median progression-free survival was 5.5 months, with a 6-month OS rate of $87.1 \%$. In cervical cancer patients, a disease control rate of $68.4 \%$ and an ORR of $26.3 \%$ have been observed after one or more systemic therapies in recurrent or metastatic settings [17]. Pembrolizumab was evaluated in recurrent metastatic cervical cancer in the Keynote 028 phase Ib trial $(n=$ 24) [18]. The Keynote 158 phase II trial $(n=98)$ showed an ORR of 17 and $12.2 \%$, respectively [16, 17]. PD-L1 expression seems to be an important predictive biomarker in this setting. While the ORR increased up to $14.6 \%$ in PD-L1 positive cancers (> 80\% of cases), no therapeutic response was seen in PD-L1 negative tumours [19]. Therefore, accelerated approval was granted for patients with advanced PD-L1 positive cervical cancer who progressed during or after chemotherapy [19]. Treatment with nivolumab is equal or superior to second or third-line chemotherapy based on the evidence from phase II trials.

In addition, methylation profiling might represent an independent modality to predict response to immune checkpoint inhibitors as recently demonstrated for lung cancer [10]. To facilitate data comparison with other cases we have included the raw methylation data as Additional file 1.

Particularly striking in comparison to the majority of (HPV-associated) cervical squamous cell carcinomas within the reference collection was the rather flat copy number profile of our case, hinting at a potential defect in DNA repair causing point mutations (not detectable with the methylation array) rather than being driven by a virus. It is, however, not entirely to be excluded that this unusual type of cancer described here evolved through infection with a rare, undetectable HPV genotype.

A strong association between TMB and response to immune checkpoint inhibitors has been reported for various cancer entities [7]. TMB and MSI-high also seem to correlate in several gynaecological cancers. However, in cervical cancer, where high TMB was seen in $6 \%$ of cases and MSI-high in $2.1 \%$ of cases, no significant correlation between both biomarkers was found [20].

Taken together, PD-L1 positivity of $30 \%$ and the high TMB (44 mutations/Mb) seem to be predictive biomarkers for response to immunotherapy and might explain the complete remission under nivolumab treatment.

As immunotherapy is used more and more frequently, it is imperative to gain insight into the management of IRAEs, which differ from classical chemotherapy side effects and might be life-threatening. The most common IRAEs affect the skin, mainly showing lichenoid 


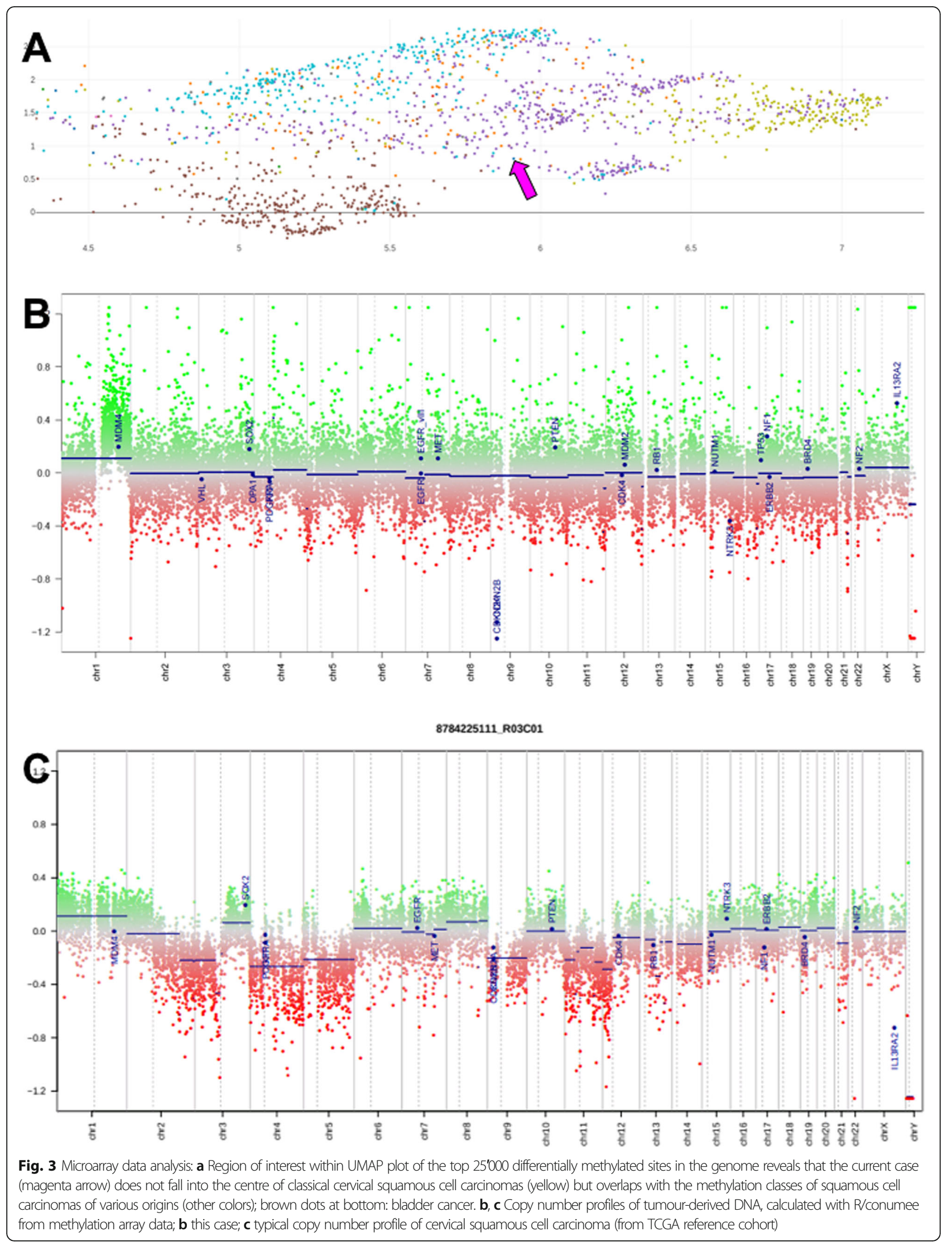


reactions, eczema, vitiligo, and pruritus. Up to $30-40 \%$ of patients under nivolumab develop cutaneous reactions [21]. Cutaneous IRAEs are generally treated with topical or systemic corticosteroids, which mostly leads to their resolution within $6-12$ weeks $[11,22]$. If symptoms are steroid-refractory, immunosuppressive agents like TNF-alpha antagonists, azathioprine or mycophenolate mofetil may be tried [21]. In the case of severe reactions, interruption of immunotherapy should be considered [11].

Isolated vulvitis has not yet been described as an IRAE. Given the broad range of potential differential diagnosis, clinicians should be aware of the importance of biopsies in determining the underlying aetiology. Early referral to a gynaeco-oncologist is crucial to ensure appropriate management. The diagnosis of immune-related vulvitis is also important to avoid the evolution of an untreated vulvopathy [22]. Although in most cases, dermatological IRAEs are manageable and reversible, the malignant potential of untreated lesions is unknown and requires further investigation.

Patients with prior radiotherapy might be more likely to develop localised and/or more severe cutaneous IRAEs in irradiated skin regions. As in our patient, the vagina had been irradiated while the vulva was outside the irradiation field, a low dose radiotherapy effect on the vulvar skin cannot be excluded. In the literature, a case of nivolumab-induced StevensJohnson syndrome with striking enhancement at the site of radiation has been reported in a patient with metastatic squamous cell carcinoma of the oropharynx [23].

The evaluation of the impact of immunotherapy and IRAEs on the quality of life is an important aspect to consider. Clinical trials in patients with advanced solid tumours showed that treatment with nivolumab causes fewer and less severe adverse events in comparison to conventional chemotherapy [25]. Nevertheless, a vulvitis as reported in our case may impact on the quality of life, sexual wellbeing and compliance of patients. Early recognition and adequate management of IRAEs are therefore essential.

\section{Conclusions}

Immunotherapy has emerged as a new option for patients with advanced cervical cancer.

We report the case of a patient with persistent complete response after third-line treatment with nivolumab for relapsed, chemotherapy-resistant cervical squamous cell carcinoma showing the promising potential of immunotherapy. Interestingly, the HPVnegative tumour was positive for PD-L1, had a very high $\mathrm{TMB}$, and a comparably flat copy number profile.
To our knowledge, this is the first report of an immunerelated vulvitis under immune checkpoint blockade. This IRAE was successfully treated with topical corticosteroids, which - by enabling nivolumab continuation - led to an excellent clinical outcome. Furthermore, we supplement our article with the tumour methylome dataset in order to provide the base for identification of comparable responders.

\section{Supplementary information}

Supplementary information accompanies this paper at https://doi.org/10. 1186/s40425-019-0742-6.

Additional file 1. IDAT methylation array data (Illumina Infinium EPIC) in zipped IDAT format (*_Grn.idat and *_Red.idat). These files are the output of the array scanner and can be used to calculate DNA methylation beta values as well as copy number profiles.

\section{Abbreviations}

AUC: Area under the curve; CT: Computer tomography; HPV: Human papillomavirus; IRAE: Immune-related adverse event; MSI-high: High levels of microsatellite instability; ORR: Objective response rate; OS: Overall survival; PD-1: Programmed cell death protein 1; PD-L1: Programmed death-ligand 1; PET-CT: Positron emission tomography-CT; RR: Response rate; TGCA: The Cancer Genome Atlas; TMB: Tumour mutational burden; UMAP: Uniform Manifold Approximation and Projection for Dimension Reduction

\section{Acknowledgements}

The authors would like to thank Tamara Ehler and Tanja Känzig for reviewing the manuscript.

\section{Authors' contributions}

We declare that all authors have made substantial contributions to the case study. FB, JH, and SF wrote the manuscript and prepared figures. CMS conceived and wrote the manuscript. TV carried out and interpreted the histopathology data. KG assisted with interpretation of histopathology data. $J \mathrm{H}$ analysed HPV test data. JH and SF performed and interpreted the methylation analysis. MB analysed and interpreted the TMB assay. MD provided the clinical follow up data. All authors reviewed, commented on and corrected the manuscript. All authors read and approved the final manuscript.

\section{Funding}

Not applicable.

\section{Availability of data and materials}

The methylome data are included as an Additional file. All other datasets obtained during workup of this case are available from the corresponding author on reasonable request.

Ethics approval and consent to participate Not applicable.

Consent for publication

Written informed consent for publication of their clinical details and clinical images was obtained from the patient.

\section{Competing interests}

The authors declare that they have no competing interests.

\section{Author details}

${ }^{1}$ Department of Gynaecology and Obstetrics, Hôpital du Jura, Delémont, Switzerland. 'Institute of Pathology, University Hospital Basel, Basel, Switzerland. ${ }^{3}$ Gynaecological Cancer Centre, University Hospital Basel and University of Basel, Basel, Switzerland. ${ }^{4}$ Department of Oncology, Hôpital du Jura, Delémont, Switzerland. ${ }^{5}$ Hospital for Women, Gynaecological Oncology, University Hospital Basel, Spitalstrasse 21, 4031 Basel, Switzerland. 
Received: 15 June 2019 Accepted: 16 September 2019

Published online: 31 October 2019

\section{References}

1. Heong V, Ngoi N, Tan DSP. Update on immune checkpoint inhibitors in gynecological cancers. J Gynecol Oncol. 2017;28(2):e20.

2. Tumeh PC, Harview CL, Yearley JH, Shintaku IP, Taylor EJM, Robert L, et al. PD-1 blockade induces responses by inhibiting adaptive immune resistance Nature. 2014;515(7528):568-71.

3. Monk BJ, Sill MW, McMeekin DS, Cohn DE, Ramondetta LM, Boardman CH, et al. Phase III trial of four cisplatin-containing doublet combinations in stage IVB, recurrent, or persistent cervical carcinoma: a gynecologic oncology group study. J Clin Oncol Off J Am Soc Clin Oncol. 2009;27(28): 4649-55.

4. Minion LE, Tewari KS. Cervical cancer - state of the science: from angiogenesis blockade to checkpoint inhibition. Gynecol Oncol. 2018;148(3): 609-21

5. Tewari KS, Sill MW, Long HJ, 3rd, Penson RT, Huang H, Ramondetta LM, et al. Improved survival with bevacizumab in advanced cervical cancer. The New England journal of medicine. 2014;370(8):734-43.

6. Liu Y, Wu L, Tong R, Yang F, Yin L, Li M, et al. PD-1/PD-L1 inhibitors in cervical Cancer. Front Pharmacol. 2019:10:65

7. Goodman AM, Kato S, Bazhenova L, Patel SP, Frampton GM, Miller V, et al. Tumor mutational burden as an independent predictor of response to immunotherapy in diverse cancers. Mol Cancer Ther. 2017;16(11):2598-608.

8. Chan TA, Yarchoan M, Jaffee E, Swanton C, Quezada SA, Stenzinger A, et al. Development of tumor mutation burden as an immunotherapy biomarker: utility for the oncology clinic. Ann Oncol Off J Eur Soc Med Oncol. 2019; 30(1):44-56.

9. Le DT, Durham JN, Smith KN, Wang H, Bartlett BR, Aulakh LK, et al. Mismatch repair deficiency predicts response of solid tumors to PD-1 blockade. Science. 2017;357(6349):409-13.

10. Duruisseaux M, Martínez-Cardús A, Calleja-Cervantes ME, Moran S. Castro de Moura M, Davalos $V$, et al. epigenetic prediction of response to anti-PD-1 treatment in non-small-cell lung cancer: a multicentre, retrospective analysis. Lancet Respir Med. 2018;6(10):771-81.

11. Haanen JBAG, Carbonnel F, Robert C, Kerr KM, Peters S, Larkin J, et al. Management of toxicities from immunotherapy: ESMO Clinical Practice Guidelines for diagnosis, treatment and follow-up. Ann Oncol Off J Eur Soc Med Oncol. 2017;28(suppl_4):iv119-42.

12. Capper D, Jones DTW, Sill M, Hovestadt V, Schrimpf D, Sturm D, et al. DNA methylation-based classification of central nervous system tumours. Nature. 2018;555(7697):469-74.

13. Mclnnes L, Healy J, Melville J. UMAP: Uniform Manifold Approximation and Projection for Dimension Reduction. ArXiv. 2018:180203426 Available from: http://arxiv.org/abs/1802.03426. Cs Stat [Internet] [cited 2019 Jun 13].

14. The Cancer Genome Atlas Research Network. Integrated genomic and molecular characterization of cervical cancer. Nature. 2017:543(7645):378-84.

15. Ojesina Al, Lichtenstein L, Freeman SS, Pedamallu CS, Imaz-Rosshandler I, Pugh TJ, et al. Landscape of genomic alterations in cervical carcinomas. Nature. 2014;506(7488):371-5.

16. Mezache L, Paniccia B, Nyinawabera A, Nuovo GJ. Enhanced expression of PD L1 in cervical intraepithelial neoplasia and cervical cancers. Mod Pathol Off J U S Can Acad Pathol Inc. 2015;28(12):1594-602.

17. Hollebecque A, Meyer T, Moore KN, Machiels J-PH, De Greve J, López-Picazo $J M$, et al. An open-label, multicohort, phase I/II study of nivolumab in patients with virus-associated tumors (CheckMate 358): Efficacy and safety in recurrent or metastatic (R/M) cervical, vaginal, and vulvar cancers. J Clin Oncol. 2017:35(15 suppl):5504

18. Frenel J-S, Le Tourneau C, O'Neil B, Ott PA, Piha-Paul SA, Gomez-Roca C, et al. Safety and Efficacy of Pembrolizumab in Advanced, Programmed Death Ligand 1-Positive Cervical Cancer: Results From the Phase Ib KEYNOTE-028 Trial. J Clin Oncol Off J Am Soc Clin Oncol. 2017;35(36):4035-41.

19. Chung HC, Ros W, Delord J-P, Perets R, Italiano A, Shapira-Frommer R, et al. Efficacy and Safety of Pembrolizumab in Previously Treated Advanced Cervical Cancer: Results From the Phase II KEYNOTE-158 Study. J Clin Oncol. 2019;37(17):1470-8.

20. Winer I, Jones NL, Xiu J, Ellerbrock A, Brown J, Herzog T. Mutational burden, tumor immune checkpoint expression, and microsatellite instability in gynecologic malignancies: implications for immune therapy. Gynecol Oncol. 2018;149:45
21. Michot JM, Bigenwald C, Champiat S, Collins M, Carbonnel F, Postel-Vinay S, et al. Immune-related adverse events with immune checkpoint blockade: a comprehensive review. Eur J Cancer. 2016;54:139-48.

22. Halonen P, Jakobsson M, Heikinheimo O, Riska A, Gissler M, Pukkala E. Lichen sclerosus and risk of cancer. Int J Cancer. 2017;140(9):1998-2002.

23. Shah KM, Rancour EA, Al-Omari A, Rahnama-Moghadam S. Striking enhancement at the site of radiation for nivolumab-induced StevensJohnson syndrome. Dermatol Online J. 2018;24(6):7

24. Shah KM, Rancour EA, Al-Omari A, Rahnama-Moghadam S. Striking enhancement at the site of radiation for nivolumab-induced StevensJohnson syndrome. Dermatol Online J. 2018 Jun 15;24(6):7.

25. Tykodi SS, Schadendorf D, Cella D, Reck M, Harrington K, Wagner S, et al. Patient-reported outcomes with nivolumab in advanced solid cancers. Cancer Treat Rev. 2018;70:75-87.

\section{Publisher's Note}

Springer Nature remains neutral with regard to jurisdictional claims in published maps and institutional affiliations.
Ready to submit your research? Choose BMC and benefit from:

- fast, convenient online submission

- thorough peer review by experienced researchers in your field

- rapid publication on acceptance

- support for research data, including large and complex data types

- gold Open Access which fosters wider collaboration and increased citations

- maximum visibility for your research: over $100 \mathrm{M}$ website views per year

At $\mathrm{BMC}$, research is always in progress.

Learn more biomedcentral.com/submission 